\title{
Identification of Errors-In-Variables Models Based on Bias Compensation of Weighted Least Squares Estimator
}

\author{
Masato Ikenoue*, Shunshoku Kanae** and Kiyoshi Wada*** \\ * National Institute of Technology, Ariake College \\ 150 Higashihagio-machi, Omuta, Fukuoka, 836-8585 Japan \\ E-mail: ikenoue@ariake-nct.ac.jp \\ ** Junshin Gakuen University \\ 1-1-1 Chikushigaoka, Minami-ku, Fukuoka, 815-8510 Japan \\ *** Professor Emeritus of Kyushu University
}

\begin{abstract}
This paper investigates the problem of identifying errors-in-variables (EIV) models, where the both input and output measurements are corrupted by white noise, and addresses a new method to solve the problem. The identification problem of EIV models with unknown noise variances has been extensively studied and several methods have been proposed. To be further developed in terms of estimation accuracy, a generalized eigenvector method with no requirement of $a$ priori knowledge about the noise variances is proposed by using the biased weighted least squares estimator. The proposed generalized eigenvalue problem can be derived by removing only the bias induced by the output noise, and thus the system parameter can be obtained as the generalized eigenvector without requiring the use of iterative identification procedure. Moreover, the bias compensation principle based algorithm, which is suitable for on-line implementation, is derived to solve the proposed generalized eigenvalue problem. The results of simulated examples indicate that the proposed approach provides good parameter estimates.
\end{abstract}

\section{Introduction}

Many identification methods are based on the assumption that input measurements are noise-free. However, this assumption is not satisfied in most practical situations. In the presence of input noise, the standard identification methods may give erroneous results [1]. Models where the both input and output measurements are corrupted by additive noises are called errors-invariables (EIV) models [2].

Several methods have been proposed to estimate unknown parameters of EIV models [2]. Koopmans-Levin method [3] is one of possible methods but it requires $a$ priori knowledge about the values of variances or the ratio to measurements noises.

On the other hand, some instrumental variable (IV) based approaches such as the eXtended IV (XIV) estimator $[4,5,6]$, the two-stage least squares (2SLS) estimator [7] and the Isermann et al.'s estimator [8] can be easily applied without any a priori knowledge of noise variances. These estimators can be viewed as the weighted least squares (WLS) estimator, and can be used in more general noise conditions by choosing an IV vector uncorrelated with noises. However, the accuracy of the parameter estimates obtained by the IV approach is often poor $[1,2,5,9]$ because this approach uses the estimates of high-lag auto-correlations and cross-correlations of the data, which yields less correlation between the IV vector and the data regression vector.

The aim of this paper is to propose a new method which does not require a priori knowledge of noise variances for the EIV models identification, where the both input and output measurements are corrupted by white noise. To be further developed in terms of estimation accuracy, the method proposed in this paper utilizes the biased WLS estimator. By allowing the IV-like vector to be correlated with only input noise, it is shown that the parameter estimation problem can be solved as the generalized eigenvalue problem which does not require a priori knowledge of noise variances. Moreover, the bias compensation based algorithm, which is suitable for on-line implementation, is derived to obtain a solution of the proposed generalized eigenvalue problem. It is demonstrated that the improvement of estimation accuracy is achieved via off-line and on-line identification. Note that the idea of removing the bias in the IV-like estimator was also used in $[5,6,10,11,12]$. So the method presented in this paper can be considered as the new application of this idea to the biased WLS estimator.

This paper organized as follows. Section 2 presents the problem statement. In section 3, the WLS estimator is briefly described and it is shown how the identification problem of the EIV models can be solved as the generalized eigenvalue problem with no requirement of a priori knowledge about the noise variances using the biased WLS estimator. Moreover, the bias compensation principle based algorithm is derived to solve the proposed generalized eigenvalue problem. The simulation results are presented in section 4 and finally section 5 gives the conclusion. 


\section{Problem Statement}

Consider the parameter estimation problem of singleinput single-output linear discrete-time system described as follows:

$$
A\left(q^{-1}\right) y_{0}(t)=B\left(q^{-1}\right) u_{0}(t)
$$

where $u_{0}(t)$ and $y_{0}(t)$ are the true input and output, $q^{-1}$ is shift operator, $q^{-1} u_{0}(t)=u_{0}(t-1)$, and the polynomials $A\left(q^{-1}\right)$ and $B\left(q^{-1}\right)$ are defined by

$$
\begin{aligned}
& A\left(q^{-1}\right)=1+a_{1} q^{-1}+\cdots+a_{n} q^{-n} \\
& B\left(q^{-1}\right)=b_{0}+b_{1} q^{-1}+\cdots+b_{n} q^{-n} .
\end{aligned}
$$

Let $u(t)$ and $y(t)$ be the noise-corrupted measurements of $u_{0}(t)$ and $y_{0}(t)$, respectively, i.e.

$$
\begin{aligned}
& u(t)=u_{0}(t)+\tilde{u}(t) \\
& y(t)=y_{0}(t)+\tilde{y}(t)
\end{aligned}
$$

where $\tilde{u}(t)$ and $\tilde{y}(t)$ are the input and output measurement noise, respectively.

The assumptions for the system, the measurement noises and the true input are made as follows:

A1. It is assumed that $A(z)$ has all zeros outside the unit circle, i.e. system is stable.

A2. It is assumed that $A\left(q^{-1}\right)$ and $B\left(q^{-1}\right)$ have no common factors.

A3. The degree of the polynomials $n$ is assumed as $a$ priori known.

A4. The input noise $\tilde{u}(t)$ and the output noise $\tilde{y}(t)$ are assumed to be mutually uncorrelated zero-mean white noises with unknown variances $\sigma_{\tilde{u}}^{2}$ and $\sigma_{\tilde{y}}^{2}$, i.e.

$$
\begin{gathered}
E[\tilde{u}(j)]=0, E[\tilde{u}(j) \tilde{u}(k)]=\sigma_{\tilde{u}}^{2} \delta_{j, k} \\
E[\tilde{y}(j)]=0, E[\tilde{y}(j) \tilde{y}(k)]=\sigma_{\tilde{y}}^{2} \delta_{j, k} \\
E[\tilde{u}(j) \tilde{y}(k)]=0, \text { for all } j, k
\end{gathered}
$$

where $E[\cdot]$ stands for mathematical expectation and $\delta_{j, k}$ is Kronecker's delta.

A5. The true input $u_{0}(t)$ is zero-mean ergodic process, and $u_{0}(t), \tilde{u}(t)$ and $\tilde{y}(t)$ are assumed to be statistically independent of each other.

To keep the notation compact in the sequel, the following notation is used

$$
\boldsymbol{M}=[\boldsymbol{m}(1) \boldsymbol{m}(2) \cdots \boldsymbol{m}(N)]^{T} \in \mathbb{R}^{N \times(n+1)}
$$

where

$$
\boldsymbol{m}(t)=[m(t) m(t-1) \cdots m(t-n)]^{T}
$$

$m(t)$ is a scalar and $N$ is the available data length. Let $\boldsymbol{y}(t), \boldsymbol{u}(t), \boldsymbol{y}_{0}(t), \boldsymbol{u}_{0}(t), \tilde{\boldsymbol{y}}(t)$ and $\tilde{\boldsymbol{u}}(t)$ be the vectors replaced $m$ in the vector $\boldsymbol{m}(t)$ by $y, u, y_{0}, u_{0}, \tilde{y}$ and $\tilde{u}$, respectively. Then (1), (2) and (3) can be written as

$$
\begin{gathered}
\boldsymbol{y}_{0}^{T}(t) \boldsymbol{a}=\boldsymbol{u}_{0}^{T}(t) \boldsymbol{b} \\
\boldsymbol{u}(t)=\boldsymbol{u}_{0}(t)+\tilde{\boldsymbol{u}}(t) \\
\boldsymbol{y}(t)=\boldsymbol{y}_{0}(t)+\tilde{\boldsymbol{y}}(t)
\end{gathered}
$$

where

$$
\begin{aligned}
\boldsymbol{a} & =\left[\begin{array}{llll}
1 & a_{1} & \cdots & a_{n}
\end{array}\right]^{T} \\
\boldsymbol{b} & =\left[\begin{array}{llll}
b_{0} & b_{1} & \cdots & b_{n}
\end{array}\right]^{T} .
\end{aligned}
$$

Moreover, let $\boldsymbol{Y}, \boldsymbol{U}, \boldsymbol{Y}_{0}, \boldsymbol{U}_{0}, \tilde{\boldsymbol{Y}}$ and $\widetilde{\boldsymbol{U}}$ be the matrices replaced $\boldsymbol{m}$ in the matrix $\boldsymbol{M}$ by $\boldsymbol{y}, \boldsymbol{u}, \boldsymbol{y}_{0}, \boldsymbol{u}_{0}, \tilde{\boldsymbol{y}}$ and $\tilde{\boldsymbol{u}}$, respectively. Now defining some matrices as

$$
\begin{aligned}
\boldsymbol{\Phi} & =\left[\begin{array}{ll}
-\boldsymbol{Y} & \boldsymbol{U}
\end{array}\right] \\
\boldsymbol{\Phi}_{0} & =\left[\begin{array}{ll}
-\boldsymbol{Y}_{0} & \boldsymbol{U}_{0}
\end{array}\right] \\
\widetilde{\boldsymbol{\Phi}} & =\left[\begin{array}{ll}
-\widetilde{\boldsymbol{Y}} & \widetilde{\boldsymbol{U}}
\end{array}\right]
\end{aligned}
$$

then (4), (5) and (6) can be written as

$$
\begin{gathered}
\boldsymbol{\Phi}_{0} \boldsymbol{\Theta}=\mathbf{0}_{N} \\
\boldsymbol{\Phi}=\boldsymbol{\Phi}_{0}+\widetilde{\boldsymbol{\Phi}}
\end{gathered}
$$

where $\mathbf{0}_{N} \in \mathbb{R}^{N \times 1}$ is a zero vector and

$$
\boldsymbol{\Theta}=\left[\begin{array}{ll}
\boldsymbol{a}^{T} & \boldsymbol{b}^{T}
\end{array}\right]^{T}=\left[\begin{array}{ll}
1 & \boldsymbol{\theta}^{T}
\end{array}\right]^{T}
$$

is the system parameter. Substituting (8) into (7) yields

$$
\Phi \Theta=-v
$$

where

$$
\boldsymbol{v}=\left[\begin{array}{llll}
v(1) & v(2) & \cdots & v(N)
\end{array}\right]^{T}=-\widetilde{\boldsymbol{\Phi}} \boldsymbol{\Theta}
$$

and $v(k)$ is a composite noise defined by

$$
v(t)=\tilde{\boldsymbol{y}}^{T}(t) \boldsymbol{a}-\tilde{\boldsymbol{u}}^{T}(t) \boldsymbol{b} .
$$

The system introduced here is usually called errors-invariables (EIV) models [2]. The problem under consideration is to estimate the system parameter $\boldsymbol{\Theta}$ from the available noise-corrupted input-output measurements $\{u(t), y(t)\}_{t=1}^{N}$, assuming $\sigma_{\tilde{u}}^{2}$ and $\sigma_{\tilde{y}}^{2}$ are unknown.

\section{Proposed Method}

\subsection{Weighted Least Squares Estimator}

Let the equation error for an estimate of $\boldsymbol{\Theta}$ be defined as

$$
\xi(t)=\boldsymbol{y}^{T}(t) \widehat{\boldsymbol{a}}-\boldsymbol{u}^{T}(t) \widehat{\boldsymbol{b}}
$$

where

$$
\widehat{\boldsymbol{\Theta}}=\left[\widehat{\boldsymbol{a}}^{T} \widehat{\boldsymbol{b}}^{T}\right]^{T}=\left[1 \widehat{\boldsymbol{\theta}}^{T}\right]^{T}
$$


is the estimate of $\boldsymbol{\Theta}$. Then the following relation can be obtained as

$$
\begin{aligned}
\boldsymbol{\xi} & =\left[\begin{array}{llll}
\xi(1) & \xi(2) & \cdots & \xi(N)
\end{array}\right]^{T} \\
& =\boldsymbol{Y} \widehat{\boldsymbol{a}}-\boldsymbol{U} \widehat{\boldsymbol{b}}=-\boldsymbol{\Phi} \widehat{\boldsymbol{\Theta}}=\boldsymbol{\omega}-\boldsymbol{\Omega} \widehat{\boldsymbol{\theta}}
\end{aligned}
$$

where

$$
\boldsymbol{\Phi}=[-\boldsymbol{\omega} \Omega]
$$

$\boldsymbol{\omega} \in \mathbb{R}^{N \times 1}$ and $\boldsymbol{\Omega} \in \mathbb{R}^{N \times(2 n+1)}$. Introduce an instrumental variable (IV) vector $\boldsymbol{z}(t)$ of dimension $n_{z} \geq$ $2 n+1$, and define the matrix

$$
\boldsymbol{Z}=\left[\begin{array}{llll}
\boldsymbol{z}(1) & \boldsymbol{z}(2) & \cdots & \boldsymbol{z}(N)
\end{array}\right]^{T} \in \mathbb{R}^{N \times n_{z}} .
$$

Consider the estimator defined by the following equation

$$
\boldsymbol{Z}^{T} \boldsymbol{\xi}=\boldsymbol{Z}^{T}(\boldsymbol{\omega}-\boldsymbol{\Omega} \widehat{\boldsymbol{\theta}})=\mathbf{0}_{n_{z}}
$$

Since the vector $\boldsymbol{z}(t)$ has higher dimension than $2 n+1$, (10) gives an overdetermined system and has no exact solution. Solving (10) in a least squares sense yields

$$
\boldsymbol{\Omega}^{T} \boldsymbol{Z} \boldsymbol{W} \boldsymbol{Z}^{T} \boldsymbol{\Omega} \widehat{\boldsymbol{\theta}}=\boldsymbol{\Omega}^{T} \boldsymbol{Z} \boldsymbol{W} \boldsymbol{Z}^{T} \boldsymbol{\omega}
$$

where $\boldsymbol{W} \in \mathbb{R}^{n_{z} \times n_{z}}$ is a positive definite weighting matrix. Thus the following estimate can be ontained if $\boldsymbol{\Omega}^{T} \boldsymbol{Z} \boldsymbol{W} \boldsymbol{Z}^{T} \boldsymbol{\Omega}$ is nonsingular.

$$
\widehat{\boldsymbol{\theta}}_{\mathrm{wls}}=\left(\boldsymbol{\Omega}^{T} \boldsymbol{Z} \boldsymbol{W} \boldsymbol{Z}^{T} \boldsymbol{\Omega}\right)^{-1} \boldsymbol{\Omega}^{T} \boldsymbol{Z} \boldsymbol{W} \boldsymbol{Z}^{T} \boldsymbol{\omega} .
$$

The estimator $\widehat{\boldsymbol{\theta}}_{\text {wls }}$ can be viewed as the weighted least squares (WLS) estimator with non-diagonal weight matrix

$$
\boldsymbol{Q}=\boldsymbol{Z} \boldsymbol{W} \boldsymbol{Z}^{T}
$$

The estimator $\widehat{\boldsymbol{\theta}}_{\text {wls }}$ corresponds to the two-stage least squares (2SLS) estimator [7] when

$$
\boldsymbol{W}=\left(\boldsymbol{Z}^{T} \boldsymbol{Z}\right)^{-1}
$$

and corresponds to Isermann et al.'s estimator [8] when

$$
\boldsymbol{W}=\boldsymbol{I}_{n_{z}}
$$

where $\boldsymbol{I}_{n_{z}} \in \mathbb{R}^{n_{z} \times n_{z}}$ is an identity matrix.

If the vector $\boldsymbol{z}(t)$ is uncorrelated with the composite noise $v(t)$, the estimator $\widehat{\boldsymbol{\theta}}_{\text {wls }}$ becomes a consistent estimate of $\boldsymbol{\theta}$. For example, the following vector $\left(n_{z}=3 n+3\right)$ can be used to remove the bias induced by the input and output noises.

$$
\boldsymbol{z}(t)=\left[\boldsymbol{y}^{T}(t-n-1) \boldsymbol{y}^{T}(t+n+1) \boldsymbol{u}^{T}(t-n-1)\right]^{T} .
$$

However, defining the vector $\boldsymbol{z}(t)$ uncorrelated with the composite noise $v(t)$ often leads to poor accuracy of the estimated parameter [1, 2, 5, 9] as also shown in simulation results. To avoid this problem, defining the vector $\boldsymbol{z}(t)$ correlated with the composite noise is required, while the estimator $\widehat{\boldsymbol{\theta}}_{\text {wls }}$ has a bias asymptotically. We denote this type of IV vector as "IV-like" vector. In the section 3.2 , by allowing the IV-like vector to be correlated with only the input noise, it is shown that the parameter estimation problem can be solved as the generalized eigenvalue problem with no requirement of a priori knowledge about the noise variances.

\subsection{Generalized eigenvalue problem}

Now let the equation error for the estimator $\widehat{\boldsymbol{\theta}}_{\text {wls }}$ be defined as

$$
\boldsymbol{\xi}_{\mathrm{wls}}=\omega-\boldsymbol{\Omega} \widehat{\boldsymbol{\theta}}_{\mathrm{wls}}
$$

then

$$
\begin{array}{r}
\boldsymbol{\Omega}^{T} \boldsymbol{Q} \boldsymbol{\xi}_{\mathrm{wls}}=\boldsymbol{\Omega}^{T} \boldsymbol{Q}\left(\boldsymbol{\omega}-\boldsymbol{\Omega} \widehat{\boldsymbol{\theta}}_{\mathrm{wls}}\right)=\mathbf{0}_{2 n+1} \\
s_{\mathrm{wls}}=\boldsymbol{\xi}_{\mathrm{wls}}^{T} \boldsymbol{Q} \boldsymbol{\xi}_{\mathrm{wls}}=\boldsymbol{\omega}^{T} \boldsymbol{Q}\left(\boldsymbol{\omega}-\boldsymbol{\Omega} \widehat{\boldsymbol{\theta}}_{\mathrm{wls}}\right)
\end{array}
$$

thus the following equation can be obtained.

$$
\left[\begin{array}{c}
-\boldsymbol{\omega}^{T} \\
\boldsymbol{\Omega}^{T}
\end{array}\right] \boldsymbol{Q}[-\boldsymbol{\omega} \boldsymbol{\Omega}]\left[\begin{array}{c}
1 \\
\widehat{\boldsymbol{\theta}}_{\mathrm{wls}}
\end{array}\right]=\left[\begin{array}{c}
s_{\mathrm{wls}} \\
\mathbf{0}_{2 n+1}
\end{array}\right] .
$$

From (9), the following relation can be obtained.

$$
\boldsymbol{\omega}=\boldsymbol{\Omega} \boldsymbol{\theta}+\boldsymbol{v} .
$$

Substituting (18) into (15) and (16) yields

$$
\begin{gathered}
\boldsymbol{\Omega}^{T} \boldsymbol{Q} \boldsymbol{\Omega} \widehat{\boldsymbol{\theta}}_{\mathrm{wls}}=\boldsymbol{\Omega}^{T} \boldsymbol{Q} \boldsymbol{\Omega} \boldsymbol{\theta}+\boldsymbol{\Omega}^{T} \boldsymbol{Q} \boldsymbol{v} \\
s_{\mathrm{wls}}=\boldsymbol{\omega}^{T} \boldsymbol{Q} \boldsymbol{\Omega} \boldsymbol{\theta}+\boldsymbol{\omega}^{T} \boldsymbol{Q} \boldsymbol{v}-\boldsymbol{\omega}^{T} \boldsymbol{Q} \boldsymbol{\Omega} \widehat{\boldsymbol{\theta}}_{\mathrm{wls}}
\end{gathered}
$$

and substituting (19) and (20) into (17) yields

$$
\left[\begin{array}{c}
-\boldsymbol{\omega}^{T} \\
\boldsymbol{\Omega}^{T}
\end{array}\right] \boldsymbol{Q}[-\boldsymbol{\omega} \boldsymbol{\Omega}]\left[\begin{array}{l}
1 \\
\boldsymbol{\theta}
\end{array}\right]=-\left[\begin{array}{c}
-\boldsymbol{\omega}^{T} \\
\boldsymbol{\Omega}^{T}
\end{array}\right] \boldsymbol{Q} \boldsymbol{v} .
$$

To remove only the bias induced by the output noise, the following IV-like vector $\left(n_{z}=3 n+3\right)$ is used instead of (14).

$$
\boldsymbol{z}(t)=\left[\boldsymbol{y}^{T}(t-n-1) \boldsymbol{y}^{T}(t+n+1) \boldsymbol{u}^{T}(t)\right]^{T} .
$$

Using the assumption A4 and A5 yields

$$
\begin{aligned}
-\operatorname{plim}_{N \rightarrow \infty} \frac{1}{N} \boldsymbol{Z} \boldsymbol{v} & =\operatorname{plim}_{N \rightarrow \infty} \frac{1}{N} \boldsymbol{Z} \widetilde{\boldsymbol{\Phi}} \boldsymbol{\Theta} \\
& =\left[\begin{array}{ll}
\boldsymbol{O}_{n+1} & \boldsymbol{O}_{n+1} \\
\boldsymbol{O}_{n+1} & \boldsymbol{O}_{n+1} \\
\boldsymbol{O}_{n+1} & \sigma_{\tilde{u}}^{2} \boldsymbol{I}_{n+1}
\end{array}\right] \boldsymbol{\Theta}
\end{aligned}
$$

where $\boldsymbol{O}_{n+1} \in \mathbb{R}^{(n+1) \times(n+1)}$ is a zero matrix. Therefore, taking probability limit of (21) yields

$$
\begin{aligned}
& \underset{N \rightarrow \infty}{\operatorname{plim}} \frac{1}{N}\left[\begin{array}{c}
-\boldsymbol{\omega}^{T} \\
\boldsymbol{\Omega}^{T}
\end{array}\right] \boldsymbol{Q}[-\boldsymbol{\omega} \boldsymbol{\Omega}] \boldsymbol{\Theta} \\
& \quad=\operatorname{plim}_{N \rightarrow \infty}\left[\begin{array}{c}
-\boldsymbol{\omega}^{T} \\
\boldsymbol{\Omega}^{T}
\end{array}\right] \boldsymbol{Z} \boldsymbol{W}\left[\begin{array}{ll}
\boldsymbol{O}_{n+1} & \boldsymbol{O}_{n+1} \\
\boldsymbol{O}_{n+1} & \boldsymbol{O}_{n+1} \\
\boldsymbol{O}_{n+1} & \sigma_{\tilde{u}}^{2} \boldsymbol{I}_{n+1}
\end{array}\right] \boldsymbol{\Theta}
\end{aligned}
$$


and thus $\Theta$ can be estimated by solving the following equation

$$
\begin{aligned}
& {\left[\begin{array}{c}
-\boldsymbol{\omega}^{T} \\
\boldsymbol{\Omega}^{T}
\end{array}\right] \boldsymbol{Q}[-\boldsymbol{\omega} \boldsymbol{\Omega}] \widehat{\boldsymbol{\Theta}}} \\
& \quad=N \sigma_{\tilde{u}}^{2}\left[\begin{array}{c}
-\boldsymbol{\omega}^{T} \\
\boldsymbol{\Omega}^{T}
\end{array}\right] \boldsymbol{Z} \boldsymbol{W}\left[\begin{array}{ll}
\boldsymbol{O}_{n+1} & \boldsymbol{O}_{n+1} \\
\boldsymbol{O}_{n+1} & \boldsymbol{O}_{n+1} \\
\boldsymbol{O}_{n+1} & \boldsymbol{I}_{n+1}
\end{array}\right] \widehat{\boldsymbol{\Theta}} .
\end{aligned}
$$

Hence (23) becomes the following generalized eigenvalue problem

$$
\boldsymbol{A} \widehat{\Theta}_{\mathrm{gev}}=\lambda_{\mathrm{gev}} \boldsymbol{B} \widehat{\Theta}_{\mathrm{gev}}
$$

where

$$
\begin{aligned}
& \boldsymbol{A}=\left[\begin{array}{c}
-\boldsymbol{\omega}^{T} \\
\boldsymbol{\Omega}^{T}
\end{array}\right] \boldsymbol{Q}[-\boldsymbol{\omega} \boldsymbol{\Omega}] \\
& \boldsymbol{B}=\left[\begin{array}{c}
-\boldsymbol{\omega}^{T} \\
\boldsymbol{\Omega}^{T}
\end{array}\right] \boldsymbol{Z} \boldsymbol{W}\left[\begin{array}{ll}
\boldsymbol{O}_{n+1} & \boldsymbol{O}_{n+1} \\
\boldsymbol{O}_{n+1} & \boldsymbol{O}_{n+1} \\
\boldsymbol{O}_{n+1} & \boldsymbol{I}_{n+1}
\end{array}\right]
\end{aligned}
$$

and

$$
\widehat{\boldsymbol{\Theta}}_{\mathrm{gev}}=\left[\begin{array}{ll}
\widehat{\boldsymbol{a}}_{\mathrm{gev}}^{T} & \widehat{\boldsymbol{b}}_{\mathrm{gev}}^{T}
\end{array}\right]^{T}=\left[1 \widehat{\boldsymbol{\theta}}_{\mathrm{gev}}^{T}\right]^{T}
$$

is the generalized eigenvector which corresponds to the smallest generalized eigenvalue $\lambda_{\text {gev }}$. Moreover, $\lambda_{\text {gev }}$ becomes

$$
\operatorname{plim}_{N \rightarrow \infty} \frac{\lambda_{\text {gev }}}{N}=\sigma_{\tilde{u}}^{2}
$$

so the input noise variance can be estimated by

$$
\widehat{\sigma}_{\tilde{u}}=\frac{\lambda_{\text {gev }}}{N} .
$$

The generalized eigenvalue problem (24) does not require a priori knowledge of noise variances and the system parameter can be obtained as the generalized eigenvector without requiring the use of iterative identification procedure. However, the generalized eigenvalue/eigenvector decomposition is not suitable for online implementation due to its high complexity and high computational power requirement. In the section 3.3, the bias compensation principle [13] based algorithm, which is suitable for on-line implementation, is derived to solve (24) without the generalized eigenvalue/eigenvector decomposition.

\subsection{Bias compensation algorithm}

Equation (23) can be written as

$$
\begin{aligned}
& {\left[\begin{array}{cc}
\boldsymbol{\omega}^{T} \boldsymbol{Q} \boldsymbol{\omega} & -\boldsymbol{\omega}^{T} \boldsymbol{Q} \boldsymbol{\Omega} \\
-\boldsymbol{\Omega}^{T} \boldsymbol{Q} \boldsymbol{\omega} & \boldsymbol{\Omega}^{T} \boldsymbol{Q} \boldsymbol{\Omega}
\end{array}\right]\left[\begin{array}{c}
1 \\
\widehat{\boldsymbol{\theta}}
\end{array}\right]} \\
& \quad=N \sigma_{\tilde{u}}^{2}\left[\begin{array}{c}
-\boldsymbol{\omega}^{T} \\
\boldsymbol{\Omega}^{T}
\end{array}\right] \boldsymbol{Z} \boldsymbol{W}\left[\begin{array}{l}
\boldsymbol{O}_{n+1} \\
\boldsymbol{O}_{n+1} \\
\boldsymbol{I}_{n+1}
\end{array}\right] \widehat{\boldsymbol{b}} .
\end{aligned}
$$

Thus the following equation can be obtained as

$$
-\boldsymbol{\Omega}^{T} \boldsymbol{Q}(\boldsymbol{\omega}-\boldsymbol{\Omega} \widehat{\boldsymbol{\theta}})=N \sigma_{\tilde{u}}^{2} \boldsymbol{\Omega}^{T} \boldsymbol{Z} \boldsymbol{W}\left[\begin{array}{c}
\boldsymbol{O}_{n+1} \\
\boldsymbol{O}_{n+1} \\
\boldsymbol{I}_{n+1}
\end{array}\right] \widehat{\boldsymbol{b}}
$$

and the following relation can be obtained if $\Omega^{T} \boldsymbol{Q} \Omega$ is nonsingular.

$$
\widehat{\boldsymbol{\theta}}=\widehat{\boldsymbol{\theta}}_{\mathrm{wls}}+N \sigma_{\tilde{u}}^{2}\left(\boldsymbol{\Omega}^{T} \boldsymbol{Q} \boldsymbol{\Omega}\right)^{-1} \boldsymbol{\Omega}^{T} \boldsymbol{Z} \boldsymbol{W}\left[\begin{array}{c}
\boldsymbol{O}_{n+1} \\
\boldsymbol{O}_{n+1} \\
\boldsymbol{I}_{n+1}
\end{array}\right] \widehat{\boldsymbol{b}} .
$$

From this relation, it can be expected that a solution of (24) can be obtained by compensating for asymptotic bias in the WLS estimator $\widehat{\boldsymbol{\theta}}_{\text {wls }}$. Hence the parameter estimate at time step $N$ is given by

$$
\widehat{\boldsymbol{\theta}}_{\mathrm{gev}}^{N}=\widehat{\boldsymbol{\theta}}_{\mathrm{wls}}+N \sigma_{\tilde{u}}^{2}\left(\boldsymbol{\Omega}^{T} \boldsymbol{Q} \boldsymbol{\Omega}\right)^{-1} \boldsymbol{\Omega}^{T} \boldsymbol{Z} \boldsymbol{W}\left[\begin{array}{c}
\boldsymbol{O}_{n+1} \\
\boldsymbol{O}_{n+1} \\
\boldsymbol{I}_{n+1}
\end{array}\right] \widehat{\boldsymbol{b}}_{\mathrm{gev}}^{N-1}
$$

where $\widehat{\boldsymbol{\theta}}_{\text {gev }}^{N}$ denotes the estimate of $\boldsymbol{\theta}$ at time step $N$.

Moreover, substituting (18) into (16) and using (15) yield

$$
\begin{aligned}
s_{\mathrm{wls}} & =(\boldsymbol{\Omega} \boldsymbol{\theta}+\boldsymbol{v})^{T} \boldsymbol{Q}\left(\boldsymbol{\omega}-\boldsymbol{\Omega} \widehat{\boldsymbol{\theta}}_{\mathrm{wls}}\right) \\
& =\boldsymbol{v}^{T} \boldsymbol{Q}\left(\boldsymbol{\omega}-\boldsymbol{\Omega} \widehat{\boldsymbol{\theta}}_{\mathrm{wls}}\right)=\left(\boldsymbol{\omega}-\boldsymbol{\Omega} \widehat{\boldsymbol{\theta}}_{\mathrm{wls}}\right)^{T} \boldsymbol{Q} \boldsymbol{v} \\
& =-\left[1 \widehat{\boldsymbol{\theta}}_{\mathrm{wls}}^{T}\right]\left[\begin{array}{c}
-\boldsymbol{\omega}^{T} \\
\boldsymbol{\Omega}^{T}
\end{array}\right] \boldsymbol{Z}^{T} \boldsymbol{W} \boldsymbol{Z} \boldsymbol{v}
\end{aligned}
$$

and taking probability limit of $s_{\text {wls }} / N$ yields

$\operatorname{plim}_{N \rightarrow \infty} \frac{s_{\mathrm{wls}}}{N}=\operatorname{plim}_{N \rightarrow \infty}\left[1 \widehat{\boldsymbol{\theta}}_{\mathrm{wls}}^{T}\right]\left[\begin{array}{c}-\boldsymbol{\omega}^{T} \\ \boldsymbol{\Omega}^{T}\end{array}\right] \boldsymbol{Z}^{T} \boldsymbol{W}\left[\begin{array}{c}\boldsymbol{O}_{n+1} \\ \boldsymbol{O}_{n+1} \\ \sigma_{\tilde{u}}^{2} \boldsymbol{I}_{n+1}\end{array}\right] \boldsymbol{b}$.

Hence the estimate of input noise variance at time step $N$ is given by

$$
\widehat{\sigma}_{\tilde{u}}^{2}=\frac{s_{\mathrm{wls}}}{N\left[1 \widehat{\boldsymbol{\theta}}_{\mathrm{wls}}^{T}\right]\left[\begin{array}{c}
-\boldsymbol{\omega}^{T} \\
\boldsymbol{\Omega}^{T}
\end{array}\right] \boldsymbol{Z}^{T} \boldsymbol{W}\left[\begin{array}{c}
\boldsymbol{O}_{n+1} \\
\boldsymbol{O}_{n+1} \\
\boldsymbol{I}_{n+1}
\end{array}\right] \widehat{\boldsymbol{b}}_{\mathrm{gev}}^{N-1}} .
$$

Based on the above discussion, the bias compensation algorithm can be established from the combination of (26) and (27).

\section{Simulation Results}

\subsection{Off-line identification}

By off-line identification, the estimates obtained by proposed generalized eigenvalue problem is compared with the least squares (LS) estimator and the two types of WLS estimators (the 2SLS estimator and the Isermann et al.'s estimator). 
Consider the following second-order system [11]:

$$
\frac{B\left(q^{-1}\right)}{A\left(q^{-1}\right)}=\frac{1.5-0.9 q^{-1}-0.45 q^{-2}}{1-0.5 q^{-1}+0.3 q^{-2}}
$$

The noise free input $u_{0}(t)$ is generated as $u_{0}(t)=$ $0.7 u_{0}(t-1)+\epsilon(t)-0.3 \epsilon(t-1)$, where $\epsilon(t)$ is a zero-mean white noise with variance $\sigma_{\epsilon}^{2}=1$. The noise variance on input side is set as $\sigma_{\tilde{u}}^{2}=0.1314$ which yields $\mathrm{SNR}=$ $10 \log _{10}\left(E\left[u_{0}^{2}(t)\right] / E\left[\tilde{u}^{2}(t)\right]\right)=10[\mathrm{~dB}]$, and the noise variance on output side is set as $\sigma_{\tilde{y}}^{2}=0.3212$ which yields $\mathrm{SNR}=10 \log _{10}\left(E\left[y_{0}^{2}(t)\right] / E\left[\tilde{y}^{2}(t)\right]\right)=10[\mathrm{~dB}]$.

The IV vector defined in (14) is used for the 2SLS estimator and the Isermann et al.'s estimator, and the IV-like vector defined in $(22)$ is used for the proposed method. Moreover, two types of biased WLS estimators are used in the proposed method. One uses the matrix $\boldsymbol{W}=\left(\boldsymbol{Z}^{T} \boldsymbol{Z}\right)^{-1}$, which is defined in (12), i.e. the biased 2SLS estimator, and we denote this method as proposed method 1. The other uses the matrix $\boldsymbol{W}=\boldsymbol{I}_{n_{z}}$, which is defined in (13), i.e. the biased Isermann et al.'s estimator, and we denote this method as proposed method 2.

Computer simulation for comparison are carried out through $M=500$ independent runs. The estimation accuracy of parameter estimates is measured in terms of the normalized root mean squared error (RMSE) which is defined by

$$
\mathrm{RMSE}=\sqrt{\frac{1}{M} \sum_{k=1}^{M} \frac{\left\|\boldsymbol{\theta}-\widehat{\boldsymbol{\theta}}^{k}\right\|^{2}}{\|\boldsymbol{\theta}\|^{2}}}
$$

where $\widehat{\boldsymbol{\theta}}^{k}$ denotes the estimates of $\boldsymbol{\theta}$ at $k$ th independent run. The mean values and the standard deviations of parameter estimates, together with the estimates of input noise variance obtained by (25) and the RMSE values, are shown in Table 1 for two different data lengths, $N=2000$ and $N=8000$.

The observations from the simulation results are as follows:

1. The LS method gives biased results.

2. The WLS estimates of $a_{1}$ and $a_{2}$ obtained by the 2SLS estimator and the Isermann et al.'s estimator are reasonably accurate. However, the accuracy of $b_{0}, b_{1}$ and $b_{2}$ are very poor even if the data length is increased.

3. The proposed method can give better results than the WLS method. The estimates of input noise variance are also close to true value. Moreover, the standard deviations of estimates and the RMSE values are being decreased in cases of larger lengths $N$. We can see that the proposed method 1 can be improved in terms of estimation accuracy of the 2SLS estimator and the proposed method 2 can be improved in terms of estimation accuracy of Isermann et al.'s estimator.
Table 1: True values, mean values and standard deviations of estimates and RMSE.

$N=2000$
\begin{tabular}{|l|r|r|c|r|r|}
\hline $\begin{array}{c}\text { True } \\
\text { value }\end{array}$ & $\begin{array}{c}\text { LS } \\
\text { estimator }\end{array}$ & $\begin{array}{c}2 \text { SLS } \\
\text { estimator }\end{array}$ & $\begin{array}{c}\text { Isermann et al.'s } \\
\text { estimator }\end{array}$ & $\begin{array}{c}\text { Proposed } \\
\text { method 1 }\end{array}$ & $\begin{array}{r}\text { Proposed } \\
\text { method 2 }\end{array}$ \\
\hline \hline$a_{1}=$ & -0.3153 & -0.4921 & -0.4989 & -0.4997 & -0.4999 \\
-0.5000 & \pm 0.0166 & \pm 0.0501 & \pm 0.0555 & \pm 0.0264 & \pm 0.0269 \\
\hline$a_{2}=$ & 0.2017 & 0.3003 & 0.3008 & 0.2995 & 0.2996 \\
0.3000 & \pm 0.0145 & \pm 0.0219 & \pm 0.0223 & \pm 0.0233 & \pm 0.0224 \\
\hline$b_{0}=$ & 1.2881 & 1.4870 & 1.4948 & 1.5159 & 1.5121 \\
1.5000 & \pm 0.0174 & \pm 0.0657 & \pm 0.0708 & \pm 0.0393 & \pm 0.0378 \\
\hline$b_{1}=$ & -0.5225 & -0.8606 & -0.8884 & -0.9108 & -0.9089 \\
-0.9000 & \pm 0.0275 & \pm 0.2147 & \pm 0.2428 & \pm 0.0539 & \pm 0.0539 \\
\hline$b_{2}=$ & -0.6011 & -0.4839 & -0.4586 & -0.4541 & -0.4519 \\
-0.4500 & \pm 0.0259 & \pm 0.1997 & \pm 0.2274 & \pm 0.0494 & \pm 0.0490 \\
\hline$\sigma_{\tilde{u}}^{2}=$ & - & - & - & 0.1383 & 0.1423 \\
0.1314 & - & - & & \pm 0.0199 & \pm 0.0205 \\
\hline RMSE & 0.2667 & 0.1633 & 0.1820 & 0.0486 & 0.0476 \\
\hline
\end{tabular}

$N=8000$
\begin{tabular}{|l|r|r|c|r|r|}
\hline $\begin{array}{l}\text { True } \\
\text { value }\end{array}$ & $\begin{array}{c}\text { LS } \\
\text { estimator }\end{array}$ & $\begin{array}{c}\text { 2SLS } \\
\text { estimator }\end{array}$ & $\begin{array}{c}\text { Isermann et al.'s } \\
\text { estimator }\end{array}$ & $\begin{array}{c}\text { Proposed } \\
\text { method 1 }\end{array}$ & $\begin{array}{c}\text { Proposed } \\
\text { method 2 }\end{array}$ \\
\hline \hline$a_{1}=$ & -0.3144 & -0.4964 & -0.4994 & -0.4994 & -0.4996 \\
-0.5000 & \pm 0.0081 & \pm 0.0285 & \pm 0.0321 & \pm 0.0130 & \pm 0.0135 \\
\hline$a_{2}=$ & 0.2014 & 0.3003 & 0.3004 & 0.2996 & 0.2998 \\
0.3000 & \pm 0.0074 & \pm 0.0113 & \pm 0.0112 & \pm 0.0121 & \pm 0.0118 \\
\hline$b_{0}=$ & 1.2871 & 1.4947 & 1.4978 & 1.5036 & 1.5023 \\
1.5000 & \pm 0.0091 & \pm 0.0351 & \pm 0.0381 & \pm 0.0207 & \pm 0.0195 \\
\hline$b_{1}=$ & -0.5214 & -0.8850 & -0.8971 & -0.9018 & -0.9017 \\
-0.9000 & \pm 0.0135 & \pm 0.1216 & \pm 0.1372 & \pm 0.0274 & \pm 0.0286 \\
\hline$b_{2}=$ & -0.6008 & -0.4631 & -0.4519 & -0.4516 & -0.4506 \\
-0.4500 & \pm 0.0131 & \pm 0.1136 & \pm 0.1297 & \pm 0.0251 & \pm 0.0249 \\
\hline$\sigma_{\tilde{u}}^{2}=$ & - & - & - & 0.1333 & 0.1342 \\
0.1314 & - & - & & \pm 0.0108 & \pm 0.0104 \\
\hline RMSE & 0.2666 & 0.0916 & 0.1030 & 0.0244 & 0.0244 \\
\hline
\end{tabular}

\subsection{On-line identification}

The proposed bias compensation alogorithm is implemented for on-line identification. The system to be identified, the true input, the input noise variance and the output noise variance are used same as in section 4.1 .

Computer simulation for comparison are carried out through $M=100$ independent runs with a data length of $N=8000$. Figure 1 gives a plot of $\operatorname{RMSE}(t)$ which is defined by

$$
\operatorname{RMSE}(t)=20 \log _{10} \sqrt{\frac{1}{M} \sum_{k=1}^{M} \frac{\left\|\boldsymbol{\theta}-\widehat{\boldsymbol{\theta}}^{k, t}\right\|^{2}}{\|\boldsymbol{\theta}\|^{2}}}[\mathrm{~dB}]
$$

where $\widehat{\boldsymbol{\theta}}^{k, t}$ denotes the estimates of $\boldsymbol{\theta}$ at time step $t$ in the $k$ th independent run. The mean values of the estimates of input noise variance obtained by (27) are shown in Fig. 2.

Similar to the results of off-line identification, simulation results indicate that the proposed method can give better results than the WLS method. The RMSE $(t)$ values obtained by the proposed bias compensation algorithm show better asymptotic property than those obtained by the WLS estimators, and the estimates of input noise variance are also close to true value. From this simulation results, we can see that the proposed bias compensation algorithm can be used for online implementation instead of the generalzied eigenvalue/eigenvector decomposition. 

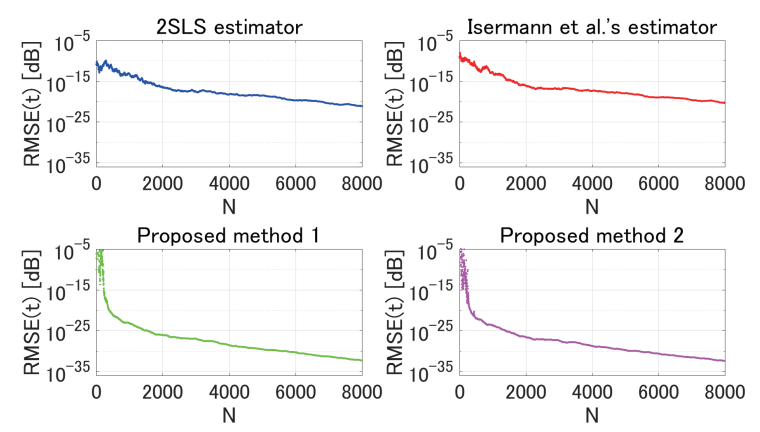

Fig. 1: $\operatorname{RMSE}(t)$ of parameter estimates.
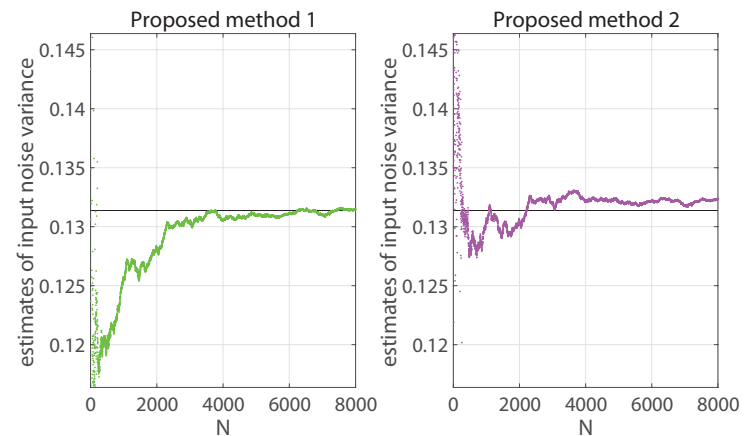

Fig. 2: The mean values of the estimates of $\sigma_{\tilde{u}}^{2}$.

\section{Conclusion}

In this paper, the method to solve identification problem of EIV models with unknown noise variances has been studied. A new method which does not require $a$ priori knowledge of noise variances has been proposed by using the biased WLS estimator. Moreover, the bias compensation principle based algorithm, which can give a solution of the proposed generalized eigenvalue problem, has been derived for on-line implementation. It has been demonstrated that the proposed method can improve in terms of estimation accuracy of the WLS estimator via off-line and on-line identification.

The proposed approach has the potential to be more improved in terms of estimation accuracy by choosing the IV-like vector $\boldsymbol{z}(t)$ and the matrix $\boldsymbol{W}$. So the way to determine these values optimally could be the topic for further research.

\section{Acknowledgement}

This work was supported by JSPS KAKENHI Grant Number JP16K18127.

\section{References}

[1] T. Söderström: Identification of stochastic linear systems in presence of input noise, Automatica, Vol. 17, No. 5, pp.713-725, 1981.
[2] T. Söderström: Errors-in-variables methods in system identification, Automatica, Vol. 43, No. 6, pp.939-958, 2007.

[3] K. V. Fernando and H. Nicholson: Identification of linear systems with input and output noise: the Koopmans-Levin method, IEE Proc. Control Theory and Appl., Vol. 132, pp.30-36, 1985.

[4] T. Söderström and K. Mahata: On instrumental variable and total least squares approaches for identification of noisy systems, Int. J. Control, Vol. 75, No. 6, pp.381-389, 2002.

[5] S. Thil, M. Gilson and H. Garnier: On instrumental variable-based methods for errors-in-variables model identification, Prep. of the 17th IFAC World Congress, Seoul, Korea, pp.426-431, 2008.

[6] S. Thil and M. Gilson: Survey of analytical IV estimates for errors-in-variables model identification, Prep. of the 18th IFAC World Congress, Milan, Italy, 2011.

[7] Ph. De Larminat: An exact recursive 2sls version of the instrumental variable estimator. Stability analysis, Proc. of American Control Conference (ACC), pp.1105-1106, 1984.

[8] R. Isermann and U. Bauer: Two-step process identification with correlation analysis and least squares parameter estimation, Trans. ASME, J. Dyn. Sys., Meas., Control, pp.426-432, 1974.

[9] K. Mahata and T. Söderström: Identification of dynamic errors-in-variables model using prefiltered data, Proc. of the 15th IFAC World Congress, Barcelona, Spain, 2002.

[10] M. Ikenoue, S. Kanae, Z. J. Yang and K. Wada: Identification of errors-in-variables models from quantized input-output measurements via biascompensated instrumental variable type method, Int. J. Innov. Comput. Inf. Control (IJICIC), vol.6, no.1, pp.183-198, 2010.

[11] R. Diversi and U. Soverini: Identification of errorsin-variables models as a quadratic eigenvalue problem, Proc. of the 12th European Control Conference (ECC), Zürich, Switzerland, pp.1896-1901, 2013.

[12] M. Ikenoue and K. Wada: Generalized eigenvector method for errors-in-variables models identification, Proc. of 54th IEEE Conf. on Decision and Control, Osaka, Japan, pp.777-782, 2015.

[13] S. Sagara and K. Wada: On-line modified leastsquares parameter estimation on linear discrete dynamic systems, Int. J. Control, Vol. 25, No. 3, pp.329-343, 1977. 